\title{
STRUCTURE OF CARBON TETRACHLORIDE $+t$-BUTANOL MIXTURES FROM POSITRON ANNIHILATION AND ULTRASONIC VELOCITY MEASUREMENTS
}

\author{
K. JERIE; A. BARANOWSKI \\ Institute of Experimental Physics, University of Wroclaw \\ pl. Maxa Borna 9, 50-204 Wroclaw, Poland \\ AND J. GLIŃSKI \\ Institute of Chemistry, University of Wroctaw \\ F. Joliot-Curie 14, 50-383 Wrocław, Poland
}

\begin{abstract}
The densities of and sound velocities in $t$-butyl alcohol solutions in $\mathrm{CCl}_{4}$ were determined in temperature range 25 to $45^{\circ} \mathrm{C}$. From these data the adiabatic compressibility coefficients of the solutions were calculated, as well as excess densities and compressibilities. The positron annihilation spectrum for this system in room temperature was measured. The results plotted against alcohol concentration suggest that the structural processes occurring in this system are caused by dimerization of the solute molecules.
\end{abstract}

PACS numbers: 61.25.Em, 78.70.Bj

\section{Introduction}

Ultrasonic velocity measurements are an useful tool for investigating liquid structure and molecular interactions in liquids. This method has been applied to pure liquids as well as to liquid mixtures of polar and non-polar components and to electrolytic solutions. Recently we compared the ultrasonic results of aqueous solutions of non-electrolytes (amines and alcohols) with those from positron annihilation experiments (see for example [1-4)).

It was found that both these different techniques applied led to consistent results which were explicable within the framework of the theory of formation of liquid pseudoclathrate structures. The successful correlation of these two experimental methods was an attempt to compare the results obtained from them for non-aqueous systems.

The local structure of a liquid mixture depends on the forces of molecular interactions and on the volume and shape of the molecules. This structure reflects 
in the thermodynamic properties of the liquid and should be expressed exactly in terms of the radial distribution function. Thus, the problem of the relation between ultrasound velocity and liquid structure could be solved exactly by means of statistical thermodynamics.

An approximate but satisfactory representation of liquid state properties was proposed by Flory [5] and applied to the interpretation of thermodynamic properties of mixtures of small non-polar molecules by Abe and Flory [6]. However, most of the authors dealing with the dependence of ultrasonic velocity, compressibility, and molar volume on the composition of binary mixtures have interpreted the experimental results phenomenologically, i.e. information about the structure has been obtained from the properties of the pure components.

This work was aimed at the relation between compressibility coefficient (calculated from ultrasonic velocity and density) and the structure of associated liquid. We chose $t$-butanol as the polar associated component which was gradually diluted with a non-polar liquid (carbon tetrachloride). Additionally, the positron annihilation measurements were done to compare with the ultrasonic ones.

Although there is a lack of data concerning the structure of liquid $t$-butanol, it is expected to form dimers in liquid state. This is supported by Raman linewidth [7] and X-ray diffraction studies [8] in $t$-butyl alcohol solutions as well as the non-linear dielectric effect in pure $t$-butanol [9].

\section{Experimental}

\subsection{Materials}

Carbon tetrachloride (pure for synth., POCh, Gliwice, Poland) was fractionally distilled and middle fraction collected.

$t$-butanol (analytical grade, Ubichem, U.K.) was distilled under reduced pressure and stored over molecular sieve to preserve absorbing water from atmosphere. Weighed portions of liquids were mixed before measuring.

\subsection{Sound velocity measurements}

The ultrasonic velocity was measured with a sing-around equipment SA-1000 constructed in the Institute of Fundamental Technical Research of the Polish Academy of Sciences, Warsaw, Poland, according to principles described by Greenspan and Tschiegg [10] and Garnsey et al. [11]. The details of our apparatus can be found in [12]. The velocity values were determined with an accuracy of about $\pm 0.05 \mathrm{~m} / \mathrm{s}$. A recording ultrathermostat (type UR, VEB MLW, Pruefgerate Medingen, GDR) was used for temperature stabilization. The temperature was stabilised with an accuracy of $\pm 0.02^{\circ} \mathrm{C}$.

\subsection{Density measurements}

Densities were measured with an accuracy of $\pm 0.5 \mathrm{~kg} \cdot \mathrm{m}^{-3}$ by weighing a calibrated sinker immersed into the liquid under test. The temperatures of density measurements were the same as in the case of sound velocity tests. 


\subsection{Positron annihilation measurements}

The lifetimes of positrons in the liquids were measured using a standard system based on the "fast-slow" coincidence technique and described in details in [1]. The annihilation experiment was performed at room temperature (approximately $\left.25^{\circ} \mathrm{C}\right)$.

\section{Experimental results}

\subsection{Acoustical measurements}

The ultrasonic velocities, as well as densities, were measured in 5 degree intervals over the temperature range $298.15-318.15 \mathrm{~K}$. The concentration dependencies of sound velocities in the solutions of $t$-butyl alcohol in $\mathrm{CCl}_{4}$, plotted against mole fraction of alcohol in the mixture, are shown in Fig. 1, showing flat minima in the region of low alcohol concentrations.

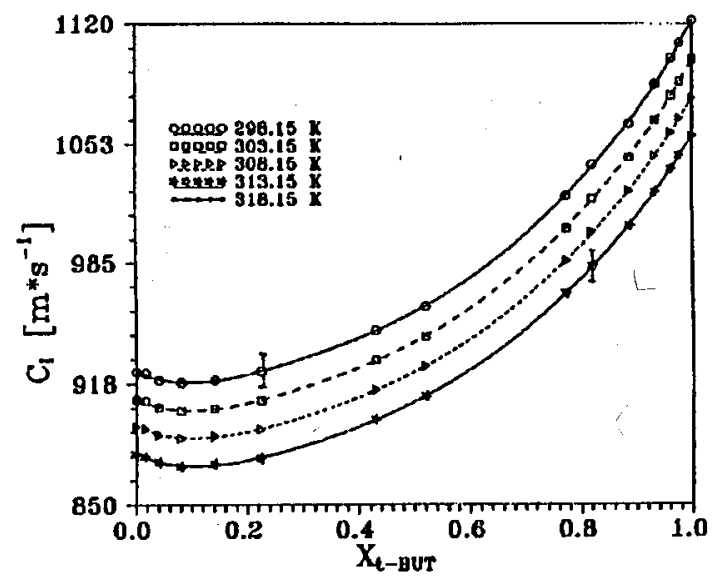

Fig. 1. The sound velocities of carbon tetrachloride $+t$-butanol mixtures vs. alcohol concentration.

From the measured densities and ultrasonic velocities, the adiabatic compressibility coefficients were calculated according to Laplace's equation

$$
\beta=\left(\rho c^{2}\right)^{-1}
$$

(where $\rho-$ density, $c-$ sound velocity). The adiabatic compressibilities plotted against the mole fraction of $t$-butanol in $\mathrm{CCl}_{4}$ are shown in Fig. 2. 


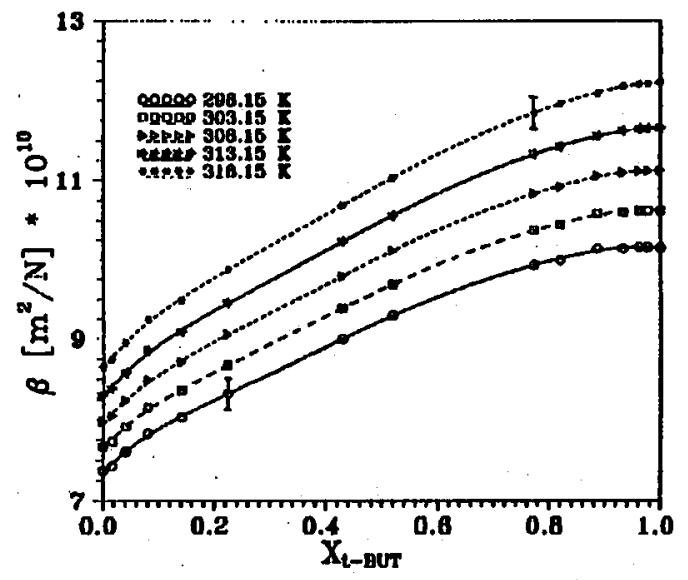

Fig. 2. The adiabatic compressibility coefficient of carbon tetrachloride $+t$-butanol mixtures vs. alcohol concentration.

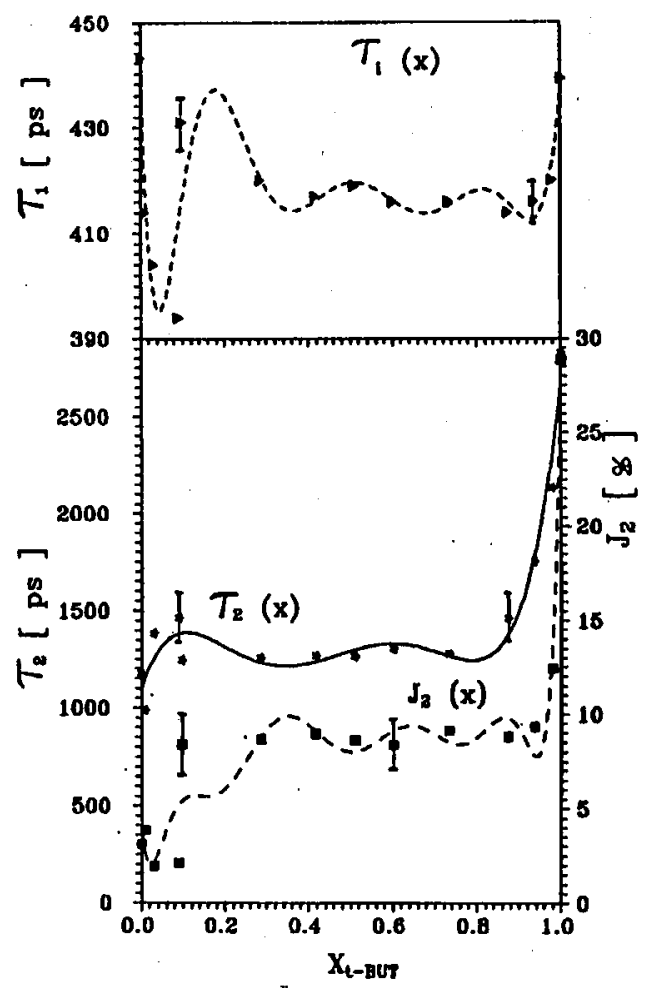

Fig. 3. The results of positron annihilation measurements vs. molar fraction of alcohol: $\tau_{1}$ - lifetimes of the short-lived component, $\tau_{2}$ - lifetimes of the long-lived component, $I_{2}$ - intensity of the latter. 


\subsection{Positron annihilation measurements}

The obtained lifetime spectra were analysed with regard to the two components $\tau_{1}$ and $\tau_{2}$ (short- and long-lived), with intensities $I_{1}$ and $I_{2}$. The short lifetime component $\tau_{1}$ of intensity $I_{1}$ is due to the free positron annihilation and to para-positronium annihilation, and the long lifetime one, $\tau_{2}$ of intensity $I_{2}$, is attributed to ortho-positronium annihilating in the pick-off process [13-15].

In this treatment the condition is fulfilled

$$
I_{1}+I_{2}=100 \% \text {. }
$$

From the analysis of the lifetime spectra of the $\mathrm{CCl}_{4}-t$-butyl alcohol mixtures the decay constants $\lambda_{1}=\left(\tau_{1}\right)^{-1}$ and $\lambda_{2}=\left(\tau_{2}\right)^{-1}$ were determined. The obtained values of $\tau_{1}, \tau_{2}$ and $I_{2}$ are presented in Fig. 3 .

The results of the annihilation experiment, when plotted against mole fraction of one of the components, show unusual character in the region of very low alcohol content in carbon tetrachloride, as well as in the region of low $\mathrm{CCl}_{4}$ content in the alcohol. From inspection of Fig. 3 it seems to be clear that the most rapid and sharp changes of the solvent structure occur when polluting it with small amounts of the other agent.

\section{Interpretation of the results}

It was already noticed [16] that deviations from the "ideal" behaviour of a liquid mixture should be useful for studying molecular interactions and structural variations. The question of the mathematical representation of the "ideality" of density, sound velocity, and compressibility of a liquid mixture was discussed in [12] on the basis of thermodynamic criterion of the ideality [17]:

$$
\bar{G}=\sum_{i} x_{i} \mu_{i}=\sum_{i} x_{i} \mu_{i}^{0}+R T \sum_{i} x_{i} \ln x_{i},
$$

where $\bar{G}$ is the mean molar free enthalpy, $x_{i}$ is the molar fraction of $i$-th component of the mixture, $\mu_{i}$ and $\mu_{i}^{0}$ are the chemical potentials of the $i$-th component in the mixture and of the pure one, respectively. The analysis of the above equation and its consequences shows that qualification of the deviations of density, sound velocity or compressibility from additivity as "excess" values seems to be thermodynamically wrong and devoided of a definite physical meaning. There was shown that the following definitions of ideal quantities are at least acceptable [12]: density -

$$
\rho^{\mathrm{id}}=\sum_{i} x_{v, i} \rho_{i}^{0}
$$

adiabatic compressibility coefficient -

$$
\beta^{\text {id }}=\sum_{i} x_{v, i} \beta_{i}^{0}
$$

(where $x_{v, i}$ - volume fraction of $i$-th component in solution, superscript 0 denotes the value for the pure component). 
Some difficulties arise [12] in defining the ultrasound velocity in an ideal mixture and we shall not use it in our interpretation. The excess densities $\rho^{\mathrm{E}}=$ $\rho-\rho^{\text {id }}$ and the excess adiabatic compressibility coefficient $\beta^{\mathrm{E}}=\beta-\beta^{\text {id }}$ were calculated for the mixtures under investigation. The results plotted against mole fraction of $t$-butanol in $\mathrm{CCl}_{4}$ are shown in Fig. 4 and Fig. 5.

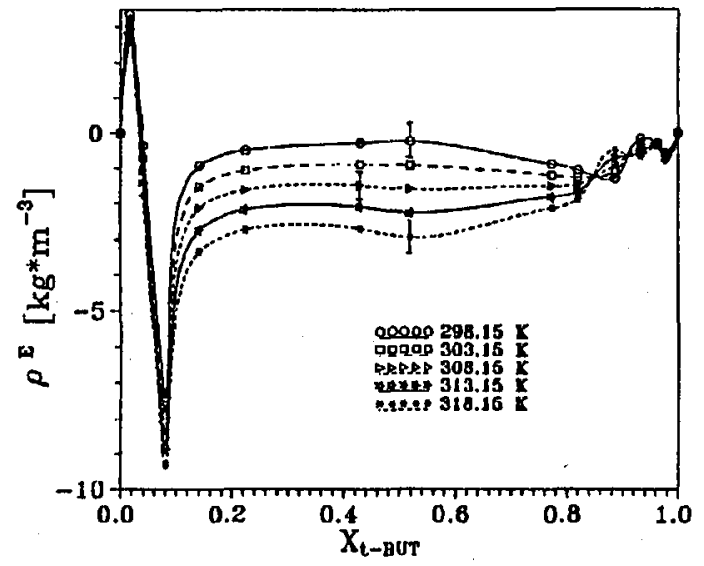

Fig. 4. The excess densities $\rho^{\mathbb{E}}$ of carbon tetrachloride $+t$-butanol mixtures vs. alcohol concentration.

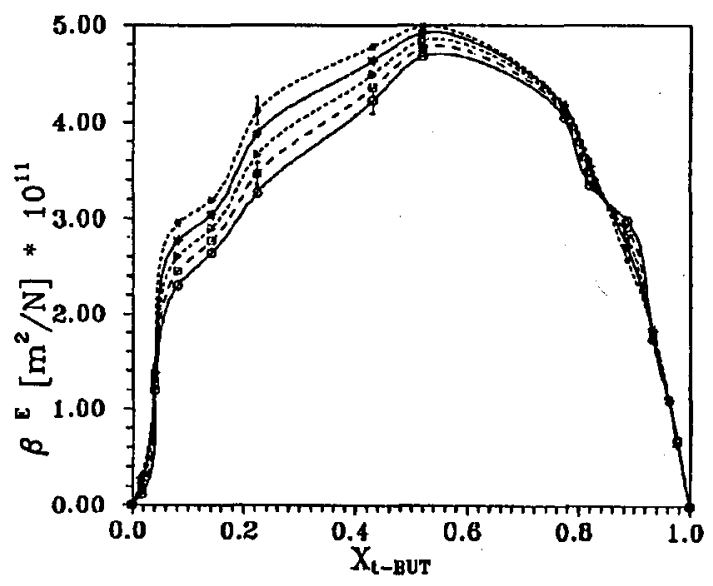

Fig. 5. The excess adiabatic compressibility coefficient $\beta^{E}$ of carbon tetrachloride + $t$-butanol mixtures vs. alcohol concentration.

The excess density seems to be much more sensitive for structural changes in liquid mixture than the excess compressibility. Consequently, the shape of curves in Fig. 4 surprisingly reminds that in Fig. 3, while the excess compressibility (Fig. 5) does not. 
Our recent papers concerning the correlation between acoustical and annihilation data in aqueous non-electrolytes show that structural changes cause fast changes of $\tau_{1}$ and $\tau_{2}$. Similarly, in the region where the changes of the liquid structure are slow (i.e. there exist definite quasi-crystalline structures), the changes of the annihilation parameters with organic solute concentration are much slower. Although any comparison between aqueous solutions of non-electrolytes and their solutions in a solvent like carbon tetrachloride is rather risky, the general idea, in our opinion, can be conserved. Taking this into consideration, the character of the changes observed in the solutions under test is as follows:

1. The region of very small alcohol concentrations in $\mathrm{CCl}_{4}$. In the range of molar fractions of alcohols from 0 to 0.1 there are observed rapid changes in both lifetimes $\left(\tau_{1}\right.$ and $\tau_{2}$ ), as well as in the long-lived component intensity $I_{2}$. The character of these changes is not clear now but surprisingly distinct and sharp. It is possible that a solvation process takes place in this region, but some other explanations are also possible (see point 3 ).

2. The region of mean alcohol concentrations. In the region of molar fractions of alcohol 0.1 to 0.9 there are no changes in excess annihilation parameters as well as in excess density (within experimental error).

3. The region of very small carbon tetrachloride concentrations. In the region of molar fraction of alcohol exceeding 0.9 there are noticeable changes in the excess annihilation parameters, but not in excess density. The most interesting, however, is the rapid increase in long-lived component of lifetime spectrum $\tau_{2}$, suggesting that this structural change is very strong. Supposing that the pure $t$-butyl alcohol in liquid state forms mainly dimers (formation of higher multimers is impeded because of the geometry of the $t$-butanol molecule), the latter process can be attributed to dimerization. However, it seems unreasonable that dimerization of $t$-butanol molecules begins at so high concentrations (i.e. $x_{t \text {-butanol }} \geq 0.9$ ). This composition corresponds to the ratio number of alcohol molecules/number of $\mathrm{CCl}_{4}$ molecules equal to (at least) $8 / 1$. The process of dimerization should start much sooner.

Although there is a lack of data concerning this system, and we feel that our results cannot solve the problem satisfactorily, the following concept is proposed. Let us assume that the dimerization process of $t$-butanol molecules begins at low alcohol concentrations (below 0.1 mole fraction). Thus, the changes in values in Figs. 3 and 4 should be attributed to the dimerization, not solvation. This idea implies the question about the nature of the process observed in high concentration range of alcohol. We are not able to answer this question. It is possible that pure (or almost pure) $t$-butyl alcohol forms in liquid state the structure more complicated than the simple mixture of dimers and monomers. To answer this question, one should investigale more than one system non-polar solvent-alcohol.

\section{Conclusions}

Concentration dependencies of the positron annihilation parameters (Fig. 3) as well as excess density and compressibility (Figs. 4 and 5) measured in the 
liquid mixtures of $t$-butyl alcohol in carbon tetrachloride show surprisingly sharp and distinct character. The changes are observed only in very low and very high alcohol concentrations. The changes in low alcohol contents were interpreted in terms of dimerization of alcohol molecules in $\mathrm{CCl}_{4}$ solution, and those in almost pure alcohol - to the formation of its own structure (for example multimerization process).

\section{Acknowledgments}

The financial support of the Commitee for Scientific Research no. PB 1168/2 is acknowledged.

\section{References}

[1] K. Jerie, A. Baranowski, B. Rozenfeld, S. Ernst, J. Gliniski, Acta Phys. Pol. A 64, 77 (1983).

[2] K. Jerie, A. Baranowski, B. Rozenfeld, S. Ernst, B. Jeżowska-Trzebiatowska, J. Gliński, Acta Phys. Pol. A 66, 3 (1984).

[3] K. Jerie, A. Baranowski, B. Rozenfeld, S. Ernst, B. Jeżowska-Trzebiatowska, J. Gliński, Acla Phys. Pol. A 66, 167 (1984).

[4] K. Jerie, A. Baranowski, S. Ernst, J. Gliński, Acta Phys. Pol. A 69, 81 (1986).

[5] P.J. Flory, J. Am. Chem. Soc. 87, 1833 (1956).

[6] A. Abe, P.J. Flory, J. Am. Chem. Soc. 87, 1838 (1956).

[7] K. Tanabe, Spectrochim. Acta A 40, 437 (1984).

[8] A.H. Narten, S.I. Sandler, J. Chem. Phys. 71, 2069 (1979).

[9] J. Małecki, S. Balanicka, J. Nowak, J. Chem. Soc. Faraday Trans. II 76, 42 (1980).

[10] M. Greenspan, C.E. Tschiegg, Rev. Sci. Instrum. 28, 897 (1957).

[11] R. Garnsey, J.R. Boe, R. Mahoney, A. Litovitz, J. Chem. Phys. 50, 5222 (1969).

[12] S. Ernst, J. Glinski, B. Jeżowska-Trzebiatowska, Acta Phys. Pol. A 55, 501 (1979).

[13] B. Levay, A. Vertes, P. Häutojarvi, J. Phys. Chem. 77, 2229 (1973).

[14] B. Levay, P. Häutojarvi, Radiochem. Radioanal. Lett. 10, 309 (1972).

[15] R.E. Wild, H.J. Ache, Radiochem. Radioanal. Lett. 23, 249 (1975).

[16] I.G. Mikhailov, N.A. Solovyev, J.P. Syrnikov, in: Osnovy molekularnoy akustiki, Nauka, Moskva 1964.

[17] I. Pirogine, R. Defay, Chemische Thermodynamik, VEB Deutscher Verlag f. Grundstoffindustrie, Leipzig 1962. 